\title{
A PROPÓSITO DE LAS ESTADÍSTICAS INESPERADAS EN DESNUTRICIÓN INFANTIL. PERFIL EPIDEMIOLÓGICO HOSPITAL CASA DEL NIÑO, CARTAGENA 2014-2015
}

\author{
Irina Suley Tirado P. ${ }^{1}$ \\ Andrea Carolina Zárate V. ${ }^{2}$
}

La desnutrición infantil tiene una serie de consecuencias negativas en varias áreas de la sociedad. Teniendo impacto en morbimortalidad, educación y productividad, constituye uno de los principales mecanismos de transmisión intergeneracional de la pobreza y la desigualdad. Por esto se decide realizar una descripción de los principales hallazgos epidemiológicos en el periodo comprendido entre enero de 2014 a diciembre de 2015 de una población constituida por pacientes atendidos en el Hospital Napoleón Franco Pareja con diagnóstico de desnutrición.

Se trata de un estudio de tipo descriptivo retrospectivo observacional, analítico de corte transversal. La población formada por 148 pacientes con diagnóstico de desnutrición clasificada en leve, moderada o severa. Las variables que se tuvieron en cuenta fueron: edad, sexo, procedencia, permanencia hospitalaria y diagnóstico. Se recolecta la información y se realiza análisis estadísticos.

Los resultados arrojaron 148 pacientes de los cuales 84 corresponden al año 2014 y 64 al 2015, menores de 18 años. Se observó que la edad más frecuente durante los dos años fue 1 año de edad, seguidamente los menores de 1 año. La mayor prevalencia fue en el sexo masculino con $60 \%$ y $55 \%$ en los años 2014-2015 respectivamente; Los departamentos relacionados de forma descendente fueron Bolívar, Sucre, La Guajira y Cesar, el promedio de estancia hospitalaria durante 2015 fue de 6,63 días con desviación estándar de 7,01.

Como se puede apreciar en la editorial titulada A pesar de las estadísticas, el número de niños muertos en Colombia por desnutrición es menor a lo esperado (Pérez, 2016), los factores de mayor impacto asociado a la desnutrición infantil y su correlación con la mortalidad infantil continúan siendo consecuencia de la pobreza extrema; Sin embargo, según las estadísticas y las políticas implementadas se evidencia una disminución en las cifras a consecuencia de la intervención en este campo.

Se concluye que la desnutrición tiene mayor prevalencia en pacientes masculinos y en menores de cinco años. A pesar de que la situación nutricional de los niños colombianos ha ido mejorando paulatinamente concordando con los resultados, esta continúa siendo una problemática actual.

\footnotetext{
1 Médica Epidemióloga, Magíster en Cuidados Paliativos Pediátricos, Centro de Investigación y Docencia Hospital Infantil Napoleón Franco Pareja, Cartagena de Indias, Bolívar. Correo: irinasuley@gmail.com - iri-29@hotmail.com

2 Médico Epidemiólogo, Residente de Primer Nivel de Cuidados Intensivos Pediátricos, Universidad de Santander.
} 


\section{Total}

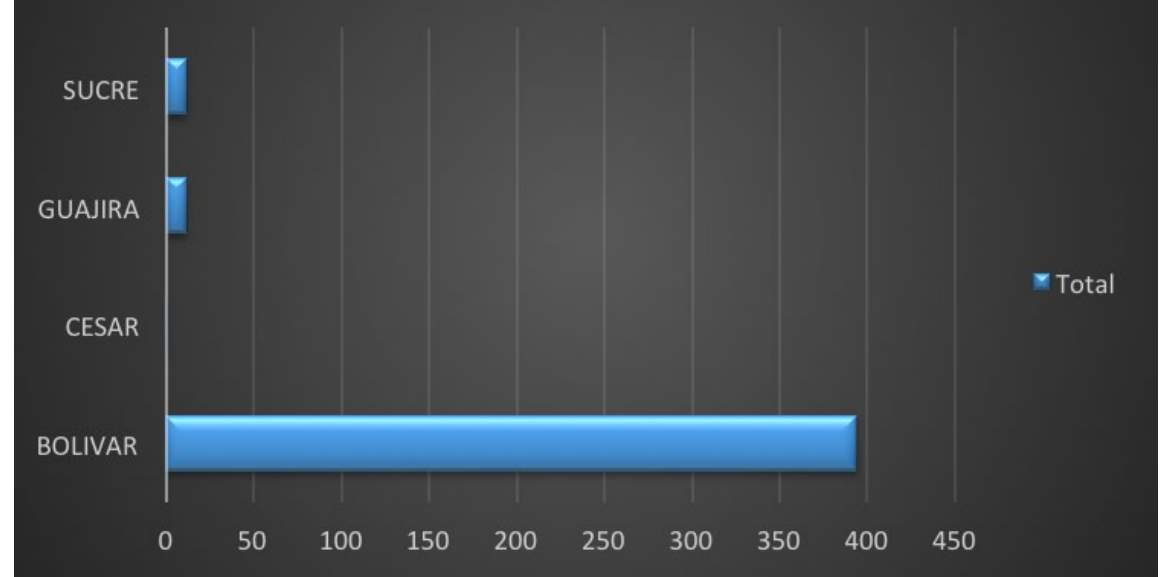

Grafica 1: Distribución según departamento de procedencia.

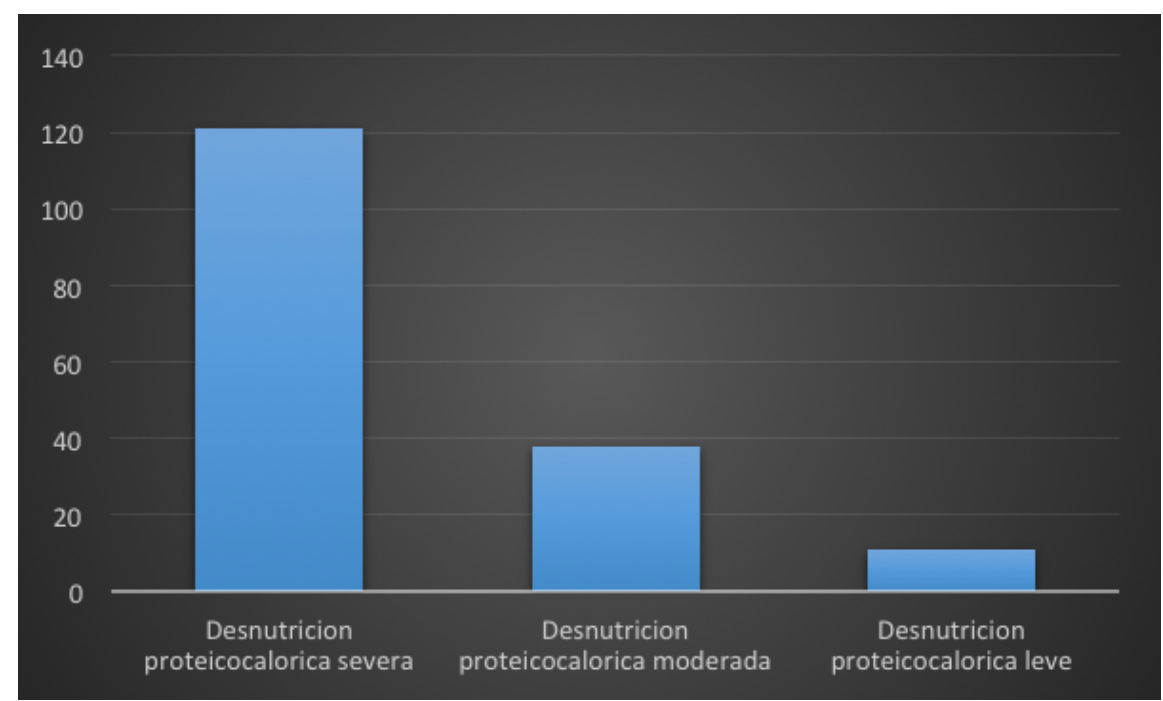

Grafica 2: Distribución según tipo de desnutrición.

\section{CONFLICTO DE INTERESES}

Todos los autores declaran no tener ningún conflicto de interés.

\section{REFERENCIAS BIBLIOGRÁFICAS}

1. Pérez-C JE. A pesar de las estadísticas el número de niños muertos en Colombia por desnutrición es menor a lo esperado [editorial]. Revista Biosalud 2016; 15(1): 5-8. DOI: 10.17151/biosa.2016.15.1.1 